\title{
Subject Index Vol. 85,1994
}

\section{Acutemyocardialinfarction 111, 397}

Age 111

Ambulatory blood pressure monitoring 101

Amlodipine 36

Amyloidosis 145

Angina pectoris 259

Angiotensin-converting enzyme inhibitor(s) 36, 137,311

Antihypertensive agents 311

- $\quad$ therapy 36

Antiphospholipid syndrome 129 Aortic regurgitation 201 Arrhythmia 361 Atherosclerosis 370

Atrial fibrillation 184

natriuretic factor 82

- peptide 334

pacing 154

Blood flow 161

- $\quad$ pressure 273

Brain natriuretic peptide 82,334

Calcium channel blockers 36

Cancer 61

Capillary permeability 161

Captopril 101

Cardiac arrhythmias 290

catheterization 94

source of embolism 53 Cardiomyopathic hamster 82 Cardiomyopathy 145 Cardiopulmonary exercise testing

341 cGMP 82 Chest pain 259 Cholesterol-fed rabbit 370 Cilazapril 311 Collagen 323 Color Doppler 129 Congestive heart failure 14,137,

235,303 Conscious dog 378

Coronary angioplasty 28, 222, 229

- $\quad$-, percutaneous transluminal

216

artery disease 171

- -, elderly 8

bypass grafting 290

heart disease, risk factors 61

reperfusion 334

sinus 154

Cranial magnetic resonance 
imaging 171 Creatine kinase 334

Diabetes mellitus 1 Diastolic function 235 Digitalis 303 Diltiazem 388 -, parenteral 290

Disopyramide 184 Dissection 229 Diuretics 311 Dobutamine 47 Doppler cardiography 235

- $\quad$ echocardiography 8,193,352

Dressler's syndrome 255

Early diagnosis 397 Echocardiography 47, 69, 129,

137 Elderly 111

- $\quad$ patients 88

Electrophysiologic study 184

Endocarditis 352

Endomyocardial biopsy 145

Endothelin 154

Enoximone 303

Etidronate 370

Exercise capacity 201

- $\quad$ stress test 407

Flow rate, calculated resistance 23 Fourier analysis 101

Gender 137 Gravitational pressure 23

Healthy normals 341 Hemodynamic significance 94 Hemodynamics 334 Hibernating

myocardium 14 Holter monitoring 28 Human heart 161 3-Hydroxy-3-methylglutaryl coen-

zyme A reductase inhibitors

244 Hypercholesterolemia 244 Hypertension 36,76,311 Hypertrophy, left ventricular 273

Inotropic drugs 303 Intramyocardial pressure 175 Ischemic episodes 28

heart disease 94

stroke 53 Isovolumic relaxation 175

Kidney 82

Lacunar infarction 171 Late potentials 216 Left atrial appendage function 121

- function 8

ventricular diastolic filling pattern 193

- - property 267

- filling 273

- function 175

- $\quad$ - systolic function 137

Lidocaine 378

Life-style 61

Long-term ECG 290

Lovastatin 370

Lyme disease 415

Magnetic resonance imaging 415 Mid-QRS potentials 216 Mitral stenosis 201 Morbidity risk

61 Mortality 111

- $\quad$ risk 61

421

Myocardial infarction 1, 222, 259, 334

-, acute 323

ischemia 154

protection 290 Myocarditis 415 Myocardium 161 
$\mathrm{Na}+, \mathrm{K}+$-ATPase 145

Nitrate therapy 28,235 Nitrendipine 101 Nitroglycerin 290 Nonvalvular atrial fibrillation 69 Norepinephrine 145

Paroxysmal supraventricular tachycardia 388

Percutaneous transluminal coronary angioplasty 88,298

Perfusion pressure 23

Peripheral nerve 145

Plaque thickness 370

Platelet aggregability 69

- $\quad$ intracellular calcium 76

Postural effects 23

Pravastatin 244

Predictors 229

Procollagen 323

Prognosis 259

Programmed stimulation 378,

388 Pulmonary artery banding 207

hypertension 207

vascular obstructive disease 207

Quantitative coronary arteriog-raphy 94

Radiofrequency catheter ablation

193 Ramipril 36 Reference values 341 Regional ejection fraction 1

- $\quad$ wall motion 14

Regression of left ventricular

hypertrophy 101 Relaxation time constant 175 Renal transplantation 273 Restenosis 222

Return to work 298 Rheumatic mitral valve disease

121 Right ventricular dysplasia 361

Serotonin 76

Silent cerebral infarction 171

- $\quad$ ischemia 407

Simvastatin 244

Spontaneous echo contrast 69

Stenosis 94

Stroke 61

Subacute myocardial infarction

378 Supraventricular tachycardia 193,

284 Symptomatic cerebrovascular

disease 171 Syndrome X 161 Systemic lupus erythematosus

129,267

Tachycardia, pediatric patients

284 99mTc-DTPA 161 Thiazide 311 Thrombolysis 111, 323 -, recombinant tissue-type plasminogen activator 255 Thrombolytic therapy 397 Total coronary occlusion 222

Transesophageal echocardiog-

raphy 53,121 Transmitral flow 267 Transstenotic pressure gradient

94 Transthoracic echocardiography

53 Transthyretin 145 Tricuspid excision 352 
- $\quad$ valve 352

Valvular replacement 201 Ventricular fibrillation 378

function 14

tachycardia 361 Ventriculoarterial coupling 1 Verapamil 175,284,388 Viscosity 76

Viscous flow pressure 23 Volume overload 175

Wavelength 184

422

Subject Index 